Opinion

\title{
Genes, Culture, and Human Evolution
}

Mark Stoneking

Department of Evolutionary Genetics, Max Planck Institute for Evolutionary Anthropology, D04103 Leipzig, Germany; E-Mail: stoneking@eva.mpg.de; Tel.: +49-341-3550502; Fax: +49-341-3550555

Academic Editor: Joep Geraedts

OBM Genetics

2017, Volume 1, Issue 2

doi:10.21926/obm.genet.1702004
Received: April 10, 2017

Accepted: May 22, 2017

Published: June 5, 2017

"I think that we've stopped evolving. Because if natural selection, as proposed by Darwin, is the main mechanism of evolution - there may be other things, but it does look as though that's the case - then we've stopped natural selection."

The above quotation, from a magazine interview, nicely summarizes the view that many people have when it comes to thinking about human evolution. Namely, humans differ from other creatures in the degree to which we rely on culture, and culture serves as an effective barrier that prevents evolution - or at least, evolution by natural selection. That is, when confronted by "problems" imposed by a change in environmental circumstances, we respond by altering our culture, not by altering our biology. To be sure, culture is not a unique human attribute; for example, tool-use by chimpanzees engaged in such activities as nut-cracking or fishing termites out of their nest meets all the standard definitions of culture, including variation among different groups and transmission between individuals by learning [1]. But no other organism relies on culture to the same extent that we do; take their nut-cracking stones or twigs for fishing out termites away from chimps and they'll make out just fine, but put one of us out in the woods without clothing, shelter, fast-food restaurants, cell phones, etc., and see how long we would last.

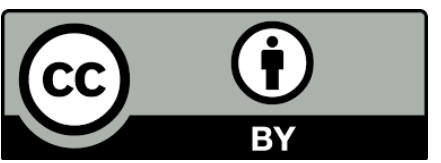

(C) 2017 by the author. This is an open access article distributed under the conditions of the Creative Commons by Attribution License, which permits unrestricted use, distribution, and reproduction in any medium or format, provided the original work is correctly cited. 
And far be it for me to try to argue that culture has not had a tremendous impact on our ability to spread and survive across more of this planet (not to mention outer space) than any other species (with the exception of our parasites). Humans didn't colonize the far northern Arctic regions by growing thicker fur, nor did they spread across the far-flung islands of the Pacific by growing gills and flippers. Obviously, cultural innovations in clothing, shelter, transportation, navigation, subsistence, etc. were responsible for the enormous success of our species. We are clearly cultural animals.

But being cultural animals does not mean that we have stopped evolving. Certainly, diseases and parasites for which we are either unable or unwilling to do what it takes to eradicate them continue to plague us, and in such instances Darwinian selection will take place - take the wellknown case of malaria resistance and sickle cell anemia as just one example. And yet at the same time, there is another aspect of culture that is receiving increasing attention, and that is the view that humans have evolved and are continuing to evolve, and we do so not just in spite of having culture, but because of culture. That is, our cultural practices can have an impact on our biological evolution, either because they directly influence variation at a particular gene, or because they have an indirect impact on our genetic variation. Moreover, if one accepts the thesis that cultural practices can impact our genetic variation, then one can turn things around and use inferences from genetics to learn more about cultural practices. I will illustrate these three points with brief examples; the reader interested in further details and/or additional examples is invited to consult my book, Introduction to Molecular Anthropology [2], from which this material is drawn.

The classic example of a cultural practice influencing the variation at a specific gene is lactase persistence (also known as lactose tolerance), which is the ability of some humans to digest lactose after weaning. Lactose is the primary sugar present in mammalian milk, and it is converted by the enzyme lactase into glucose and galactose, from which cells get most of their energy. All mammals produce lactase while nursing at their mother's breast, but most mammals stop producing lactase shortly after weaning, which makes evolutionary sense as most mammals never encounter lactose after weaning. However, some of us humans are weird in that we continue producing lactase into adulthood, and the frequency of lactase persistence is strongly correlated with dairying, not just in Europe but also in Africa and elsewhere [3]. Moreover, the patterns of genetic variation around the lactase gene indicate that the relatively high frequency of lactase persistence (up to $80-90 \%$ in some populations) that was attained over the course of just a few thousand years could only have been attained by the action of natural selection - and pretty strong natural selection at that, as this is one of the strongest signals of selection in the human genome [3,4]. However, the evolutionary benefit of drinking milk remains unresolved; various explanations have been proposed, including milk as a source of nutrition, as a source of calcium, or as a source of clean drinking water. Lactase persistence also provides a nice example of convergent evolution, as different mutations were selected for in Europe and Africa [5]. So, a cultural trait - drinking milk - has resulted in evolution via natural selection, resulting in lactase persistence.

An example of a cultural practice that has had an indirect influence on genetic variation involves residence patterns - that is, whether after marriage the woman moves to the residence of the man (patrilocality) or the man moves to the residence of the woman (matrilocality) - and the maternal vs. paternal genetic history of human populations. Humans are blessed with two types of DNA that make it possible to investigate maternal vs. paternal genetic history, and these 
are the maternally-inherited mitochondrial DNA (mtDNA) and the male-specific parts of the $Y$ chromosome (MSY). And the first comprehensive study of human mtDNA and MSY variation found that genetic differences between populations were, on average, bigger for the MSY than for mtDNA [6]. The authors considered various possible explanations, and suggested that the most likely was a higher rate of female than male migration between populations, as a consequence of the widespread practice of patrilocality. The idea is that the regular movement of females between populations results in more widespread sharing of mtDNA genomes and hence smaller genetic differences. Conversely, the tendency of males to stay put results in less sharing and movement for the MSY, and consequently larger genetic differences. An obvious test of this hypothesis is to compare mtDNA and MSY variation in matrilocal populations, and indeed a subsequent study found exactly the opposite pattern in matrilocal populations, with bigger genetic differences between populations for mtDNA than for the MSY [7]. So, differences in the movement of males vs. females between populations - due to a cultural practice, namely the socially-dictated residence pattern - has had an indirect influence on mtDNA vs. MSY variation.

As an aside, there are those who would say that the above example of residence pattern influencing patterns of mtDNA vs. MSY variation reflects trivial changes in gene frequencies; population geneticists may regard this as evolutionary change, but it is not biologically-meaningful as no selection or adaptation is involved. However, such an attitude ignores the fact that changes in gene frequencies, regardless of the cause, can have biological consequences. For example, there was a bottleneck (reduction in population size) involved in the migration of modern humans out of Africa, with additional bottlenecks involved as humans moved further and further from Africa [8]; it is becomingly clear that these serial bottlenecks have resulted in increased frequencies of deleterious alleles in non-African populations that likely have a negative impact on health [9]. Thus, non-adaptive processes such as genetic drift or other seemingly innocuous changes in allele frequencies can have biological consequences.

Finally, an example of using genetic analyses to learn more about associated cultural practices concerns dating the origin of clothing. Here, the genetic analyses are not of humans but of one of our parasites, namely lice. Lice are ubiquitous parasites across the animal world, but humans are unique in that whereas most creatures are plagued by just one kind of lice, we have three different kinds of lice. Two of these are the head louse, Pediculus capitis, and the body louse, Pediculus humanus (the third type of louse will be mentioned below). These are closely-related species that differ primarily in their ecology: the head louse lives and feeds on the human scalp, while the body louse feeds on the human body but lives and lays eggs in our clothing. Presumably, before humans started wearing clothing, we had only head lice; clothing provided a new ecological niche which lice moved into and adapted to, eventually evolving to become body lice. One can therefore use a molecular clock approach to analyze louse DNA and date the divergence of head and body lice, which by inference then indicates when clothing became important. The resulting estimate of $70,000-100,000$ years ago for the divergence of head and body lice [10] suggests a relatively recent origin of clothing, corresponding to the time when modern humans first began moving out of Africa. Thus, knowing that the cultural practice of wearing clothing has had genetic consequences (for our lice, if not for us) gives us the means of learning more about that cultural practice - namely, when clothing became important in human evolution, something that could not be discerned from the fossil or archaeological record. 
And what of the third type of louse? While not directly related to my main thesis about genes and culture, this is still an interesting story. The third type is the pubic louse (Phthiris pubis), which (as the name suggests) lives and feeds on the pubic region of humans. At first glance, it may seem natural to suppose that the differentiation between pubic lice and the ancestors of the other two lice arose when humans lost body hair. That is, prior to losing body hair, humans had one type of louse all over their body, as is the case for other apes. Then our ancestors lost body hair, the naked torso became a geographic "barrier" to the movement of lice between the head and the pubic region, and eventually via classic allopatric speciation, pubic lice and head lice evolved into separate species, with head lice later giving rise to body lice when humans started wearing clothing. However, this simple scenario contradicts the taxonomy of these lice, as human head and body lice are classified in the same genus as the chimpanzee louse (Pediculus), while pubic lice are classified in the same genus as the gorilla louse (Phthirus). And DNA analyses confirm that the taxonomy is correct; the human pubic louse is indeed more closely related to the gorilla louse than to the other human lice, and diverged from the gorilla louse about 3-4 million years ago [11]. How humans got pubic lice from gorillas remains a matter of conjecture. Still, assuming that the transfer occurred after humans lost body hair and thus the pubic region was available as a novel ecological niche, then the divergence between pubic and gorilla lice would suggest that body hair was lost relatively soon after our lineage diverged from that of our nearest relatives, chimpanzees, about 6-8 million years ago.

In conclusion, there are two sides to the definition of humans as cultural animals. Culture has unquestionably allowed humans to colonize far more of this planet than any other species, and to be far more successful than any other species, because culture freed us from the yoke of biological adaptation. At the same time, cultural practices have also influenced our genetic variation, both directly and indirectly, and continue to do so. Thus, we can use genetic analyses to learn more about associated cultural practices - as well as other interesting aspects of our evolutionary history, such as the loss of body hair, as reflected in the transfer of pubic lice from gorillas to our ancestors.

\section{Acknowledgements}

This perspective is based on a public lecture given on 5 October 2016 while I was the Eugène Dubois Chair at Maastricht University, The Netherlands. I thank Joep Geraedts for the invitation to write this perspective and numerous colleagues who have influenced my thinking on this subject.

\section{References}

1. Boesch C. Is culture a golden barrier between human and chimpanzee? Evol Anthropol. 2003;2:82-91.

2. Stoneking M. Introduction to Molecular Anthropology. Hoboken, New Jersey: John Wiley \& Sons; 2017.

3. Ingram CJ, Mulcare CA, Itan Y, Thomas MG, Swallow DM. Lactose digestion and the evolutionary genetics of lactase persistence. Hum Genet. 2009;124:579-91. 
4. Bersaglieri T, Sabeti PC, Patterson N, Vanderploeg T, Schaffner SF, Drake JA, et al. Genetic signatures of strong recent positive selection at the lactase gene. Am J Hum Genet. 2004;74:1111-20.

5. Tishkoff SA, Reed FA, Ranciaro A, Voight BF, Babbitt CC, Silverman JS, et al. Convergent adaptation of human lactase persistence in Africa and Europe. Nat Genet. 2007;39:31-40.

6. Seielstad MT, Minch E, Cavalli-Sforza LL. Genetic evidence for a higher female migration rate in humans. Nat Genet. 1998;20:278-80.

7. Oota $H$, Settheetham-Ishida W, Tiwawech D, Ishida T, Stoneking M. Human mtDNA and $\mathrm{Y}$-chromosome variation is correlated with matrilocal versus patrilocal residence. Nat Genet. 2001;29:20-1.

8. Prugnolle F, Manica A, Balloux F. Geography predicts neutral genetic diversity of human populations. Curr Biol. 2005;15:R159-60.

9. Henn BM, Botigue LR, Peischl S, Dupanloup I, Lipatov M, Maples BK, et al. Distance from sub-Saharan Africa predicts mutational load in diverse human genomes. Proc Natl Acad Sci USA. 2016;113:E440-9.

10. Kittler R, Kayser M, Stoneking M. Molecular evolution of Pediculus humanus and the origin of clothing. Curr Biol. 2003;13:1414-7.

11. Reed DL, Light JE, Allen JM, Kirchman JJ. Pair of lice lost or parasites regained: the evolutionary history of anthropoid primate lice. BMC Biol. 2007;5:7.

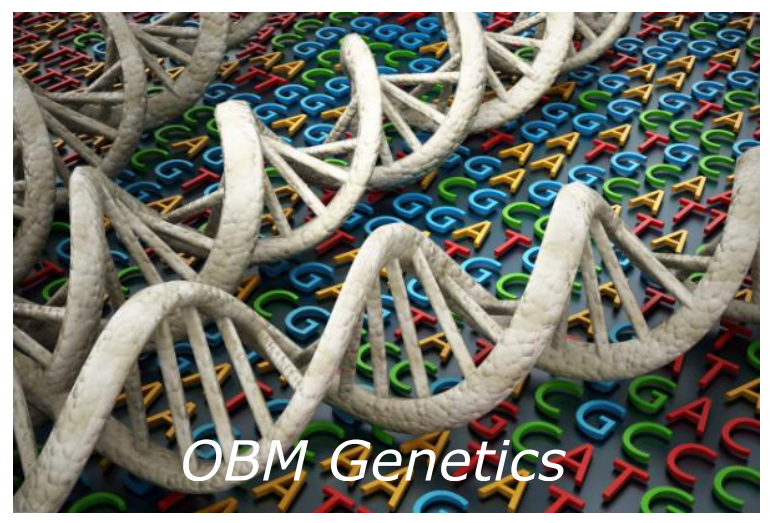

Enjoy OBM Genetics by:

1. Submitting a manuscript

2. Joining in volunteer reviewer bank

3. Joining Editorial Board

4. Guest editing a special issue

For more details, please visit: http://www.obm-pc.com/journals/genetics 